\title{
QUALIDADE DE VIDA NA PERCEPÇÃO DA EQUIPE DE ENFERMAGEM DA UNIDADE DE TERAPIA INTENSIVA: REVISÃO INTEGRATIVA
}

\section{QUALITY OF LIFE IN THE PERCEPTION OF THE NURSING TEAM OF THE INTENSIVE CARE UNIT: INTEGRATIVE REVIEW}

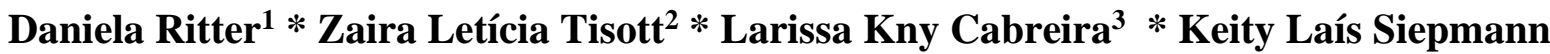 \\ Soccol $^{4}$
}

\begin{abstract}
RESUMO
A enfermagem, por vezes, possui altos índices de estresse, baixa Qualidade de Vida e insatisfação entre estes profissionais, em especial os que atuam em Unidade de Terapia Intensiva. Diante disso, o estudo tem como objetivo analisar as evidências científicas relacionadas à percepção da equipe de enfermagem que atua em Unidade de Terapia Intensiva adulto sobre qualidade de vida. Trata-se de uma pesquisa de revisão integrativa da literatura realizada em abril de 2020 nas bases de dados Literatura Latino-Americana e do Caribe em Ciências da Saúde e Base de Dados de Enfermagem e SCOPUS, ainda na Biblioteca Eletrônica Científica Online. Foram incluídos artigos originais, no período temporal dos últimos dez anos nos idiomas inglês, português e espanhol. Foram selecionados nove artigos, que resultaram em quatro categorias: a percepção da equipe de enfermagem sobre a qualidade de vida relacionada a si; a qualidade de vida relacionada à equipe de enfermagem e a qualidade de vida relacionada ao cuidado com os pacientes. A percepção dos profissionais acerca da qualidade de vida da Unidade de Terapia Intensiva fornece subsídios para a construção da Qualidade de Vida desses profissionais logo reflete em sua forma de cuidar e interagir no espaço da Unidade de Terapia Intensiva.
\end{abstract}

Palavras-chave: Enfermagem; Equipe de Enfermagem; Qualidade de Vida; Unidade de Terapia Intensiva.

\begin{abstract}
Nursing sometimes has high levels of stress, low Quality of Life and dissatisfaction among these professionals, especially those working in the Intensive Care Unit. Therefore, the study aims to analyze the scientific evidence related to the perception of the nursing staff working in an adult Intensive Care Unit about quality of life. This is an integrative literature review research carried out in April 2020 in the Latin American and Caribbean Literature in Health Sciences and Nursing and SCOPUS databases, also in the Online Scientific Electronic Library. Original articles were included, in the temporal period of the last ten years, in English, Portuguese and Spanish. Nine articles were selected, which resulted in four categories: the perception of the nursing team about the quality of life related to themselves; the quality of life related to the nursing team and the quality of life related to patient care. The perception of professionals about the quality of life in the Intensive Care Unit provides subsidies for the construction of the Quality of Life of these professionals, which soon reflects on their way of caring and interacting in the space of the Intensive Care Unit.
\end{abstract}

Keywords: Nursing; Nursing Team; Quality of Life; Intensive Care Unit.

\footnotetext{
${ }^{1}$ Universidade Regional do Noroeste do Rio Grande do Sul (UNIJUI). Ijuí/Brasil. Orcid:000-0001-9284-3244.

${ }^{2}$ Universidade Federal do Rio Grande do Sul (UFRGS). Porto Alegre/ Brasil. Orcid: 0000-0001-9489-3951.

${ }^{3}$ Universidade Regional do Noroeste do Rio Grande do Sul (UNIJUI). Brasil/RS. Orcid: 0000-0003-0099-9734.

${ }^{4}$ Universidade Franciscana (UFN). Santa Maria/ Brasil. Orcid: 0000-0002-7071-3124.
} 


\section{INTRODUÇÃO}

O trabalho é entendido como um dos pilares da vida, o qual é relevante para a satisfação pessoal, devido ao fato de gerar reconhecimento social e proporcionar identidade às pessoas. Quando executado pode torna-se um fator desgastante na vida dos trabalhadores ou gerar satisfação ${ }^{(1)}$. A Qualidade de vida (QV) corresponde à percepção do indivíduo sobre sua situação de vida, meio cultural, valores no qual está inserido, objetivos, expectativas e preocupações. Está vinculado à saúde física, estado psíquico, independência, relações sociais, crenças e influências do ambiente ${ }^{(2)}$.

$\mathrm{O}$ estresse é a forma física e emocional como o corpo reage à pressão, podendo manifestar sintomas mentais e físicos. Os efeitos do estresse são diferentes e cada um reage de uma forma diferente. $\mathrm{O}$ estresse pode prejudicar o desempenho profissional $\mathrm{e}$ pessoal e consequentemente a sua atuação frente à equipe de trabalho, afetando a equipe e toda a organização, logo a dinâmica do setor, sendo um fator importante no processo organizacional, bem como na capacidade com que seus trabalhadores lidam com o estresse $^{(3)}$.

Os profissionais de enfermagem são considerados com maior suscetibilidade para contaminação, desgastes físicos e emocionais decorrentes da profissão ${ }^{(4)}$. Pesquisa realizada com enfermeiros de uma Unidade de Terapia Intensiva (UTI) demonstrou 64,29\% dos entrevistados referirem à alta exaustão emocional, tornando o profissional de enfermagem mais suscetível e vulnerável a dores psicossomáticas, e/ou físicas, como síndrome de Burnout e estresse ${ }^{(5)}$. Além disso, o enfermeiro pode desenvolver alteração de humor, depressão, culpa e estes sentimentos são considerados estressores e podem influenciar negativamente no desenvolvimento profissional, o que poderá acarretar o aumento da ansiedade ${ }^{(6)}$.

Neste ínterim, é necessário trabalhar a qualidade de vida, satisfação e a promoção do autocuidado da equipe de enfermagem no setor da UTI com o intuito de promover a melhora física e bem-estar psicoemocional dos profissionais. Através da garantia à saúde e ao bem-estar da equipe de enfermagem, a fim de atingir a melhora e reequilíbrio dos usuários. Diante disso, o estudo tem como objetivo: analisar as evidências científicas relacionadas à percepção da equipe de enfermagem que atua em UTI adulto sobre qualidade de vida.

\section{MÉTODO}

Trata-se de uma revisão integrativa da literatura. A revisão integrativa é uma ampla abordagem metodológica, o qual tem como finalidade sintetizar resultados coletados a partir de materiais experimentais e não experimentais para uma melhor compreensão sobre um determinado tema ${ }^{(7)}$.

O desenho do estudo baseou-se em seis etapas distintas: elaboração da questão de pesquisa; busca por pares e seleção da 
amostra; definição das informações a serem extraídas dos artigos selecionados; análise das informações; interpretação dos resultados e síntese do conhecimento. Para nortear a busca, foi formulada a questão de pesquisa. A partir do acrônimo PICO $(P$ : population/patients; $I:$ intervention; $C$ : control; O: outcome), definindo-se "equipe de enfermagem" como população, "qualidade de vida" como fenômeno de interesse e "Unidade de Terapia Intensiva adulto" como contexto. Desse modo, a questão estruturada foi: quais as evidências relacionadas à percepção da equipe de enfermagem que atua em UTI adulto sobre qualidade de vida?

A coleta de dados foi realizada em abril de 2020, por dois revisores de forma independente, mediante consulta às bases de dados Literatura Latino-Americana e do Caribe em Ciências da Saúde (LILACS) e Base de Dados de Enfermagem (BDENF) via Biblioteca Virtual em Saúde. Ainda na Biblioteca Eletrônica Científica Online (SCIELO) e na base de dados SCOPUS. Para operacionalização da busca foram selecionados os descritores controlados (DC) constantes no Medical Subject Headings (MeSH) e Descritores em Ciências da Saúde (DeCS). Recorreu-se ao operador booleano AND para combinação dos seguintes termos: enfermagem and Unidade de Terapia Intensiva and Qualidade de vida conforme.

Para seleção dos artigos os critérios de inclusão foram: artigos originais, disponíveis na íntegra, nos idiomas inglês, português e espanhol, dos últimos 10 anos. Já os critérios de exclusão foram estudos que não respondessem à questão de pesquisa. Esta etapa foi realizada, a partir da leitura do título e resumo, para seleção dos artigos. Após tal seleção, foi realizada a leitura destes materiais pré-selecionados na íntegra. Os artigos que foram encontrados repetidos em mais de uma base de dados, foram considerados duplicados, lidos e selecionados apenas uma vez.

Foram identificadas, inicialmente, 901 publicações. Na base de dados da LILACS, encontrou-se a partir da pesquisa com os descritores selecionados, 73 artigos, com critérios de inclusão temporal, foram selecionados 44 artigos, sendo destes após a leitura de título e resumo foram préselecionados 13 artigos dos quais foi selecionado dois artigos após a leitura na íntegra.

Na base de dados BDENF, consistiram 57 artigos, com critérios de inclusão temporal, foram selecionados 38 artigos, com a leitura do título e resumo, após foram préselecionados 11 artigos e após a leitura na íntegra foram utilizados dois artigos. $\mathrm{Na}$ biblioteca virtual SCIELO, equivaleram-se em seleção inicial 32 artigos, foi realizada a leitura do título e resumo e pré-selecionados 10 artigos, e após a leitura na íntegra foram utilizados para esta pesquisa três artigos.

$\mathrm{Na}$ SCOPUS, com os descritores supracitados foram encontrados 739 artigos, após aplicar os três filtros: acesso livre, tipo 
de documento artigo, língua inglês, português e espanhol reduziu-se para 84 artigos disponíveis, os quais foram selecionados a partir da leitura do título e resumo selecionando 10 artigos e após ler na íntegra,

Figura 1 - Fluxograma do processo de busca, seleção e inclusão dos estudos. Ijuí, RS, 2021.

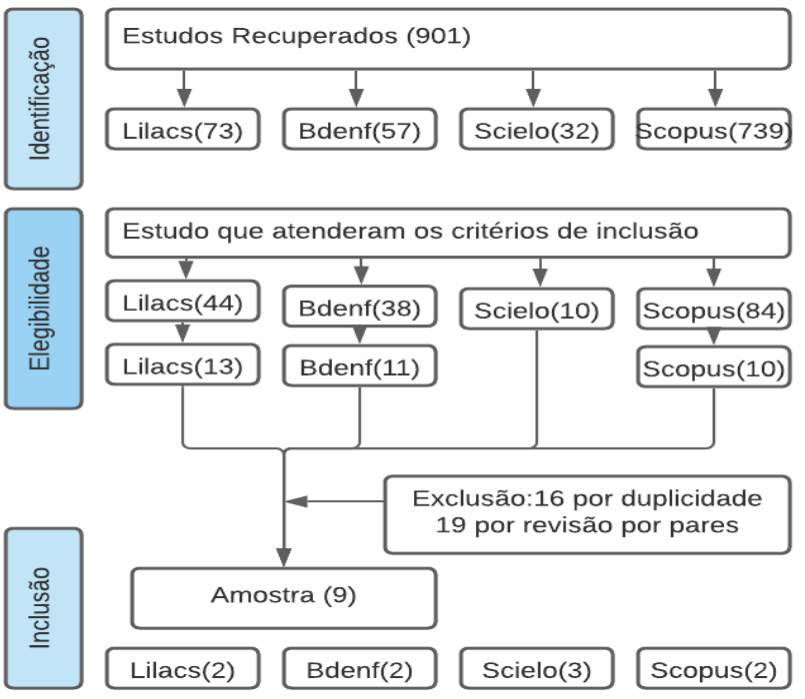

Fonte: Os autores

\section{Análise e tratamento dos dados}

Para a avaliação da qualidade metodológica dos estudos incluídos, foram utilizadas as recomendações que considera: nível 1: meta-análise de múltiplos estudos clínicos controlados e randomizados; nível 2: estudos individuais com delineamento experimental; nível 3: estudos quaseexperimentais; nível 4: estudos descritivos (não-experimentais) ou com abordagem qualitativa; nível 5: relatos de caso ou de experiência; e, nível 6: evidências pautadas na opinião de especialistas ${ }^{(8)}$. Para a etapa de análise e interpretação, foi construído um instrumento de coleta e síntese dos dados coletados pela própria autora com as foram eleitos dois artigos para a utilização nesta pesquisa. A Figura 1 apresenta o processo de busca, seleção e inclusão dos estudos. 


\section{RESULTADOS}

Para esse estudo de revisão foram selecionados nove artigos. Os quais foram publicados em oito periódicos diferentes, entre eles: Revista Brasileira de Enfermagem, Revista Mineira de Enfermagem, Revista de Enfermagem da UFSM, Actualidades Paul em Enfermagem, Neurology Psychiatry abd Brain Research, International Journal of Ocupational and Entrenmented Medicine, Investimento Educação Enfermagem, e dando destaque para a Revista de Pesquisa: Cuidado
É Fundamental Online, com dois artigos. Quanto ao ano de publicação, a maioria dos estudos selecionados concentrou-se no ano de 2013, totalizando dois artigos. Nos anos de 2007, 2011, 2012, 2014, 2017, 2018 e 2020 foi encontrado um artigo para cada respectivo ano, para o tema proposto nesta pesquisa.

Quanto aos Níveis de Evidências (NE), seis são nível IV, dois estudos são níveis II e um nível I, dando destaque a pesquisa de caráter qualitativo. A identificação dos artigos selecionadas está apresentada no Quadro 2.

Quadro 2 - Caracterização dos artigos selecionados. Ijuí, RS, 2021.

\begin{tabular}{|c|c|c|c|c|}
\hline Autores e ano & Título & Periódico & Metodologia & NE \\
\hline $\begin{array}{l}\text { 9. LEMOS; RENNÓ; PASSOS, } \\
2012\end{array}$ & $\begin{array}{l}\text { Satisfação no trabalho da enfermagem em } \\
\text { UTI. }\end{array}$ & $\begin{array}{l}\text { Cuidado é } \\
\text { Fundamental Online }\end{array}$ & $\begin{array}{l}\text { Pesquisa } \\
\text { Qualitativa }\end{array}$ & IV \\
\hline 10. RAMOS et al., 2014 & $\begin{array}{l}\text { Qualidade de Vida no Trabalho: } \\
\text { Repercussões para a saúde do trabalhador } \\
\text { de enfermagem de terapia intensiva }\end{array}$ & $\begin{array}{l}\text { Cuidado É } \\
\text { Fundamental Online }\end{array}$ & $\begin{array}{l}\text { Pesquisa } \\
\text { Qualitativa }\end{array}$ & IV \\
\hline 11. SCHIMIDT et al; 2013 & $\begin{array}{l}\text { Qualidade de Vida no trabalho e Burnout } \\
\text { em trabalhadores de enfermagem de } \\
\text { unidade de terapia intensiva }\end{array}$ & $\begin{array}{l}\text { Revista Brasileira de } \\
\text { Enfermagem }\end{array}$ & $\begin{array}{l}\text { Pesquisa } \\
\text { Descritiva } \\
\text { Qualititativa }\end{array}$ & IV \\
\hline $\begin{array}{l}\text { 12. } \quad \text { MORAES; } \\
\text { SONATI, } 2018\end{array}$ & $\begin{array}{l}\text { Percepção da Qualidade de vida de } \\
\text { profissionais de enfermagem de terapia } \\
\text { intensiva }\end{array}$ & $\begin{array}{l}\text { Revista Mineira de } \\
\text { Enfermagem }\end{array}$ & $\begin{array}{l}\text { Pesquisa } \\
\text { transversal } \\
\text { qualitativa }\end{array}$ & IV \\
\hline 13. KLEINUBING et al; 2013 & $\begin{array}{l}\text { Estresse Coping em Enfermeiros de } \\
\text { Terapia Intensiva Adulto e Cardiológica }\end{array}$ & $\begin{array}{ll}\text { Revista } & \text { de } \\
\text { Enfermagem } & \text { da } \\
\text { UFSM } & \end{array}$ & $\begin{array}{l}\text { Pesquisa } \\
\text { Quantitativo }\end{array}$ & II \\
\hline $\begin{array}{l}\text { 14. PASCHOA; } \\
\text { WHITAKER, } 2007\end{array}$ & $\begin{array}{l}\text { Qualidade de vida dos trabalhadores de } \\
\text { enfermagem de unidade terapia intensiva }\end{array}$ & $\begin{array}{l}\text { Actualidades Paul } \\
\text { em Enfermagem }\end{array}$ & $\begin{array}{l}\text { Pesquisa } \\
\text { Quantitativa }\end{array}$ & II \\
\hline 15. ELKONIN; VYVER, 2011 & $\begin{array}{l}\text { Positive and negative emotional responses } \\
\text { to work-related trauma of intensive care } \\
\text { nurses in private health care facilities }\end{array}$ & Original Research & $\begin{array}{l}\text { Pesquisa } \\
\text { Qualitativa } \\
\text { exploratório e } \\
\text { descritivo }\end{array}$ & IV \\
\hline 16. ÇELIK et al., 2017 & $\begin{array}{l}\text { Fatigue in Intensive Care Nurses and } \\
\text { Related Factors }\end{array}$ & $\begin{array}{l}\text { International Journal } \\
\text { of Ocupational and } \\
\text { Entrenmented } \\
\text { Medicine }\end{array}$ & $\begin{array}{l}\text { Pesquisa } \\
\text { Qualitativa }\end{array}$ & IV \\
\hline 17. HUANG et al., 2020 & $\begin{array}{l}\text { EFFECT of Balint Group Training on } \\
\text { burnout and quality of work life among } \\
\text { intensive care nurse: a randomized } \\
\text { controlled trial }\end{array}$ & $\begin{array}{l}\text { Neurology, } \\
\text { Psychiatry abd Brain } \\
\text { Research }\end{array}$ & $\begin{array}{l}\text { Estudo } \\
\text { Clinico } \\
\text { Randomizado }\end{array}$ & I \\
\hline
\end{tabular}

Fonte: Os autores 


\section{DISCUSSÃO}

Após leitura e releitura do material advindo da revisão bibliográfica foi possível agregar o conteúdo, por convergência de ideias, em quatro temas em que o primeiro versa sobre: a percepção da equipe de enfermagem sobre a QV relacionada a si; o segundo trata sobre a QV relacionada à equipe de enfermagem; e o terceiro diserta acerca da qualidade de vida relacionada ao cuidado com os pacientes.

\section{A percepção da equipe de enfermagem} sobre a qualidade de vida relacionada a si

Segundo os profissionais da UTI adulto a QV está vinculada a ter uma boa remuneração salarial, pois alguns estudos trazem que este fator causa desânimo e o profissional precisa ter mais de um emprego. Entre os estudos selecionados a remuneração é o maior gerador de insatisfação no trabalho, devido ao fato de não ser correspondente a reponsabilidade exigida dos profissionais de saúde, nem tampouco, a valorização salarial aos profissionais que a equipe tanto $\operatorname{almeja}^{(9,10,11,12,14)}$.

O baixo salário é um fator estressante para os profissionais de enfermagem, comparado a outras profissionais de saúde. A curva de crescimento de contratação do profissional enfermeiro tem uma tendência elevada, trabalhando a mesma jornada semanal que vários profissionais, contudo apresenta diferença salarial, o qual para o enfermeiro, na maioria das vezes, não corresponde a sua respectiva função, responsabilidade e jornada trabalhada ${ }^{(18)}$.

De acordo com estudos selecionados os profissionais trazem que a $\mathrm{QV}$ está ligada ao bem estar, vinculadas às atividades de lazer, aliada a momentos de descanso com a família e nos turnos de trabalho ${ }^{(9,14,16)}$, ligados também a um sono adequado, regular e poder ter escolha do local onde trabalhar ${ }^{(11,12,14,16)}$. A presença de trabalhadores na enfermagem está cada vez mais aumentada no turno noturno pela necessidade financeira, devido a um adicional noturno repassado a estes profissionais, entretanto trabalhar a noite está impactando negativamente o bem-estar e saúde dos trabalhadores, causando distúrbios no ritmo biológico, alterando o padrão do sono, mau relacionamento com a família, pouco contato com os filhos gerando problemas de saúde ao trabalhador, associado a possíveis erros no local de trabalho ${ }^{(19)}$.

Além disso, os artigos selecionados mostram que é importante estar bem consigo mesmo ${ }^{(10)}$, para conseguir enfrentar o contato contínuo com os sofrimentos e morte dos usuários $^{(9,11)}$. Pois a busca evidenciou que a falta de QV está ligada ao aumento do estresse no trabalho na UTI, vinculada a ansiedade, depressão, Burnout e fadiga por compaixão ${ }^{(10,13,15,16,17)}$. A UTI é considerada um dos setores mais estressantes e com alta exposição e risco para o desenvolvimento da síndrome de Burnout, o qual é evidenciado 
pela interação entre equipe de enfermagem e pacientes críticos e estar próximo da dor e sofrimento humano ${ }^{(20)}$

Além de prejuízos emocionais, os profissionais trazem a falta de qualidade de vida ligada as exaustões físicas ${ }^{(10,14,15)}$, como dores, tremores, ligadas a dependência de medicações, muitas vezes recorrem a esta medida para aliviar estes desconfortos, para a execução do serviço, e melhor qualidade da assistência $\operatorname{prestada}^{(14)}$. Estes desconfortos, relatados pelos profissionais da saúde, predominam-se as doenças infectocontagiosas e os Distúrbios Osteomusculares Relacionados ao Trabalho (DORT), os quais acarretam danos físicos e psíquicos muitas vezes desencadeados ao trabalhar em pé, levantamento de peso e posturas inadequadas.

Os DORT's acarretam altos custos, de tratamento, podendo causar disfunção e/ou incapacidade do profissional, e consequentemente reduzir sua produtividade no trabalho ${ }^{(21)}$.

Estudos selecionados trazem que os profissionais de saúde referem fazer poucas atividades físicas em seu dia a dia, os quais trazem o sedentarismo como algo prejudicial para si. Logo os profissionais entendem que possuir QV é fazer exercício físico ${ }^{(12)}$. Estudo realizado com profissionais de saúde que atuam em UTI's de cinco hospitais da região metropolitana do Recife, traz que os profissionais classificados como ativos apresentaram maiores escores de QV, nos domínios capacidade funcional, vitalidade e saúde mental quando comparados aos inativos $^{(22)}$.

$\mathrm{Na}$ percepção dos profissionais de enfermagem a QV está direcionada a aspectos relacionados a si. Assim os profissionais que atuam na UTI trazem em sua percepção que QV está relacionada a ter um bom salário, salário pelo serviço destes profissionais e respaldo para atuação da equipe de enfermagem. Além disso, trazem a qualidade do sono a realização de exercícios físicos e conciliação entre a carga trabalhada na UTI, e momentos de lazer como uma alternativa para cuidar de si e ter uma melhor QV entre a equipe de enfermagem.

\section{A qualidade de vida relacionada à equipe de enfermagem.}

A QV para os profissionais da UTI está relacionada às relações sociais entre a equipe que atua na UTI. Desse modo os artigos selecionados trazem que ter um bom relacionamento com os colegas que trabalham na assistência e também na gestão é um fator fundamental para ter harmonia no trabalho ${ }^{(9,10,11,14)}$. O bom relacionamento interpessoal entre os profissionais da enfermagem influencia positivamente, pois ocorre uma "transferência" de bons sentimentos, fazendo com que a equipe trabalhe mais alegre, e de forma mais produtiva no setor ${ }^{(23)}$.

Aliado a isso um estudo traz sobre a importância da comunicação entre a equipe de 
enfermagem como um fator positivo para a QV no trabalho( ${ }^{(9)}$ como consequência uma boa relação com o paciente. A comunicação interfere positivamente na interação da equipe de enfermagem, requer entendimento entre os envolvidos, no qual todos os envolvidos devem estar atentos a comunicação verbal e não verbal, o qual pode evitar conflitos na assistência e na satisfação da equipe ${ }^{(24)}$.

Os estudos selecionados apontam que a QV está relacionada ao reconhecimento profissional, pois os profissionais dizem que não são reconhecidos no ambiente de trabalho, não sendo respeitados pelos seus superiores (Lemos; Rennó; Passos, 2012). A valorização e reconhecimento dos profissionais de enfermagem gera um sentimento de aceitação e aprovação dentro da equipe, o qual interfere positivamente em sua saúde psíquica como indivíduo e profissional, outro fator influente é o respaldo profissional a qual interfere positivamente dando autonomia e segurança a equipe de enfermagem, nas tomadas de decisões e cuidados prestados aos pacientes ${ }^{(23)}$.

Em relação à QV no trabalho os profissionais trazem a importância do Aperfeiçoamento profissional/ aprimoramento das técnicas/ onde desejam aliar mais conhecimento teórico em sua forma de trabalhar $^{(9)}$. A qualidade de vida está relacionada ao aprendizado entre a equipe, vinculado aos desafios constantes no setor, sendo assim a equipe menciona que essa aprendizagem na equipe é fundamental para a resolutividade dos problemas ${ }^{(13)}$.

Visando manter a qualidade da assistência prestada são importantes a educação continuada e a educação permanente, as quais são essenciais para o aperfeiçoamento profissional, uma vez que, tais processos tenham como objetivo mudar a realidade do setor positivamente, com conhecimentos novos, sobre os temas relacionados à assistência prestada no setor. Este treinamento realizado de forma conjunta deve suprir as reais necessidades da unidade, para uma melhor assistência aos usuários em todos os âmbitos, prevenção, promoção, recuperação, tratamento e reabilitação ${ }^{(25)}$.

Os profissionais trazem que a $\mathrm{QV}$ relacionada a equipe de enfermagem é ter um bom relacionamento com os colegas e gestão, uma boa comunicação entre a equipe, reconhecimento profissional, sendo respeitados pelos seus superiores. Trazem ainda a importância do aperfeiçoamento profissional, aprimorando técnicas e a importância da educação continuada e permanente.

\section{A qualidade de vida relacionada ao cuidado com os pacientes}

Estudo traz a falta de materiais para cuidar do paciente, logo os profissionais relatam que para ter $\mathrm{QV}$ o profissional necessita de material adequado como pacote de curativos e higiene e aparelhos em bom 
funcionamento para realizar seu trabalho de forma eficaz oferendo confiança e segurança ao paciente. $\mathrm{O}$ uso de material adequado associado ao fato de haver recursos suficientes, é essencial para garantir proteção à equipe e aos pacientes, e assim garantir o desenvolvimento do trabalho da enfermagem para melhor assistência aos usuários, resultando em um ambiente seguro e saudável tanto para equipe quanto para os pacientes ${ }^{(10)}$. A qualidade e a quantidade de materiais na unidade influência para a continuidade de uma assistência de enfermagem qualificada, pois a falta ou má qualidade do mesmo coloca em risco, tanto os profissionais de enfermagem como as pessoas que eles $\operatorname{assistem}^{(26)}$.

Ainda, é mencionado, em estudo selecionado, que o atendimento qualificado e a resolutividade das ações com os pacientes causam importante fator de motivação e QV ao profissional de enfermagem. Tais fatos influenciam diretamente na satisfação do trabalhador, devido ao fato deste estar acompanhando e atuando na assistência, e assim observar a melhora do paciente a partir de suas ações, condutas e bom atendimento prestado pela equipe de enfermagem a estes usuários $^{(9)}$.

A resolutividade do cuidado, e demais demandas requeridas dentro da UTI, influenciam na QV. Isso ocorre, devido ao fato de os profissionais receberem um feedback positivo, por parte dos pacientes, que notam o bom desempenho e atuação destes profissionais, e com a melhora destes usuários geram prazer maior dentre a enfermagem. $\mathrm{O}$ qual, consequentemente ameniza os fatores estressantes e melhorando a QV e incentivando a atuação destes profissionais, e permitindo que os mesmos alcancem os objetivos individuais e profissionais, assim como oportuniza o crescimento do indivíduo, como pessoa e como profissional da equipe de enfermagem $^{(27)}$.

Um artigo selecionado, nessa revisão, traz que a alta complexidade no cuidado com o paciente, causa insatisfação ao profissional. $\mathrm{O}$ fato do profissional de enfermagem, estar prestando um cuidado a pacientes com grande sofrimento, risco de morte e alta complexidade geram um desgaste e insatisfação a enfermagem o qual influencia na assistência prestada aos usuários da UTI, portanto, é essencial a realização de ações gerenciais para amenizar a insatisfação decorrente desta alta complexidade e sofrimento que o profissional está exposto deste ambiente de trabalho ${ }^{(11)}$.

A assistência de enfermagem prestada ocasiona diversos benefícios, contudo, não isenta de risco. A preocupação com a prevenção de riscos está constantemente presente no cuidado aos pacientes em estado crítico internados na UTI, associado com o uso constante de tecnologia e a complexidade nos cuidados prestados. A UTI, é um dos setores que não permite erros, já que está relacionado diretamente à vida e morte dos 
pacientes. A insatisfação e prejuízo na QV do trabalhador na UTI são causados por esta pressão no ambiente de trabalho ${ }^{(28)}$.

Os profissionais trazem que a $\mathrm{QV}$ relacionada ao cuidado com os pacientes está relacionada com a necessidade de material adequado e suficiente para cada paciente, é poder prestar um bom atendimento $\mathrm{e}$ solucionar questionamentos trazendo resolutividade o mais precoce possível.

Fomento: Não se aplica

\section{CONSIDERAÇÕES FINAIS}

Através do estudo foi possível analisar as evidências científicas relacionadas à percepção da equipe de enfermagem que atua em UTI adulto referentes à qualidade de vida. Os profissionais de enfermagem percebem que a qualidade de vida ao atuarem no setor da UTI está relacionada a vários fatores. Os profissionais percebem que a $\mathrm{QV}$ está direcionada a questões que estão relacionados a si, como o fato de possuir um bom salário, conseguir estar junto a família, ter momentos de descanso, lazer e cuidados com a saúde mental e física.

Os profissionais da enfermagem percebem que a QV também está aliada as relações com a equipe de trabalho no espaço da UTI, como a comunicação, o reconhecimento enquanto profissional e o aprendizado junto aos demais colegas. No olhar da equipe a QV está relacionada ao processo de cuidar do paciente, seja com os sentimentos que desperta no profissional durante seu turno de trabalho como nos recursos disponíveis para oferecer a eles, sendo que o atendimento de qualidade e a resolutividade das ações com os pacientes causam importante fator de motivação e QV.

Esse estudo fornece subsídios para compreender a percepção da QV entre os profissionais da enfermagem. No entanto, o estudo possui limitações relacionada a produção de conhecimento envolvendo restrita a metodologias que produzem fraco nível de evidência. Logo se sugere a ampliação dos estudos realizados nessa área do conhecimento, a fim de proporcionar melhor QV aos profissionais de enfermagem da UTI, logo melhor atendimento aos pacientes e relação entre equipe.

\section{REFERÊNCIAS}

1. Silva AE, Lima, PKM, Oliveira C. (Qualidade de vida dos profissionais de enfermagem de nível médio em Unidade De Terapia Intensiva. Rev. de Enfermagem do Centro Oeste Mineiro. 2016. 6(3):231823330. Disponível em: https://www.scielo.br/scielo.php?pid=S00347 $1672013000100002 \&$ script=sci_arttext\&tlng $=\mathrm{pt}$.

2. Pretto CR, et al. Quality of life of chronic kidney patients on hemodialysis and related factors. Revista Latino-Americana Enfermagem. 2020. 28:e3327. Disponível em:

https://www.scielo.br/scielo.php?pid=S0104$11692020000100372 \&$ script=sci_arttext. 
3. Brochado C, Ribas, JLC. Estresse da equipe de enfermagem na UTI. Rev Saúde e Desenvolvimento. 2018. 13(12):44-57. Disponível em: https://www.revistasuninter.com/revistasaude/ index.php/saudeDesenvolvimento/article/view 1998.

4. Filha DML et al. Cuidamos dos outros, mas quem cuida de nós? Vulnerabilidades e implicações da covid-19 na enfermagem. Revista Enfermagem Foco. 2020. 1(11):135140. Disponível em: http://revista.cofen.gov.br/index.php/enferma gem/article/view/3521

5. Nascimento EÉF. et al. Desenvolvimento da síndrome de burnout nos enfermeiros de uti de um hospital privado do agreste pernambucano. Brazilian Journal of health Review. 2020. 3 (4):7325-7352. Disponível em:

https://www.brazilianjournals.com/index.php/ BJHR/article/view/12611.

6. Ribeiro WA, et al. Evidências e repercussões do estresse vivenciado pelos enfermeiros da unidade de terapia intensiva: um estudo das publicações brasileiras. Revista Pró-UniverSUS. 2019.10 (1): 61-65. Disponível em: http://editora.universidadedevassouras.edu.br/ index.php/RPU/article/view/1659.

7. Souza MT, Silva, MD, Carvalho, R. Revisão integrativa: o que é e como fazer. Revista Einstein. 2017. 8(1):102-106. Disponível em: https://www.scielo.br/scielo.php?pid=S167945082010000100102\&script=sci_arttext\&tlng $=\mathrm{pt}$.

8. Stetler CB. et al. Utilization-focused integrative reviews in a nursing service. Appl Nurs Res. 1998. 11(4):195-206. Disponível em:

https://www.sciencedirect.com/science/article /pii/S0897189798803297.

9. Lemos, MC, Rennó, CO, Passos, JP. Satisfação no trabalho da enfermagem em UTI. Revista de Pesquisa: Cuidado é
Fundamental Online. 2012. 4(4):2890-2900. Disponível em: https://www.redalyc.org/pdf/5057/505750895 011.pdf.

10. Ramos É. L, et al. (2014). Qualidade de vida no trabalho: repercussões para a saúde do trabalhador de enfermagem de terapia intensiva. Revista de Pesquisa: Cuidado é Fundamental Online, 6(2):571-583. Disponível em: https://www.redalyc.org/pdf/5057/505750622 013.pdf.

11. Schmidt DRC. et al. Qualidade de vida no trabalho e burnout em trabalhadores de enfermagem de Unidade de Terapia Intensiva. Revista Brasileira de Enfermagem. 2013. 66(1):7-13. Disponível em: https://www.scielo.br/scielo.php?pid=S0034$71672013000100002 \&$ script=sci_arttext\&tlng $=$ pt.

12. Moraes BFM, Martino, MMF, Sonati, JG. Percepção da qualidade de vida de profissionais de enfermagem de terapia intensiva. REME - Revista Mineira de Enfermagem. 2018. 22:e-1100. Disponível em: http://reme.org.br/artigo/detalhes/1251.

13. Kleinubing RE et al. Estresse e coping em enfermeiros de terapia intensiva adulto e cardiológica. Revista de Enfermagem da UFSM. 2013. 3(2):335-344, 2013. Disponível em:

https://periodicos.ufsm.br/index.php/reufsm/a rticle/view/8924.

14. Paschoa S, Zanei SSV, Whitaker IY. Qualidade de vida dos trabalhadores de enfermagem de terapia intensiva. Revista Atualidades Paulista de Enfermagem. 2017. 20(3):305-310. Disponível em: https://www.scielo.br/scielo.php?pid=S0103$21002007000300010 \&$ script $=$ sci_abstract $\&$ tl $\mathrm{ng}=\mathrm{pt}$.

15. Elkonin D. Vyver LVD. Positive and negative emotional responses to work-related trauma of intensive care nurses in private health care facilities. Rev Original Research. Africa do Sul. 2017. 2018;16(1):436-444. 
Disponível

em:

https://www.ajol.info/index.php/hsa/article/vi ew/70951.

16. Çelik S. et al. Fatigue in Intensive Care and Related Factors. Int $\mathrm{J}$ Occup Environ Med. 8(4). Disponível em: https://www.ncbi.nlm.nih.gov/pmc/articles/P MC6679605/.

17. Huang H. et al. Effect of balint group training on burnout and quality of work life among intensive care nurses: a randomized controlled trial. Neurology Psychiatry and Brain Research. 2020. 35(1):16-21. Disponível em: https://www.sciencedirect.com/science/article /pii/S0941950019301101?casa_token $=\mathrm{x} 0 \mathrm{w} 8 \mathrm{~F}$ 7G9x7QAAAAA:oFDP7ftYHwvhG1iRXV3J iB2rFYXRZJzi8DSrTBNY7THQmC_Lvs1s1 TEm5ZiMVOKFQbth_2xPzQ.

18. Felli VEA. Condições de trabalho de enfermagem e adoecimento: motivos para a redução de jornada de trabalho para 30 horas. Enfermagem em Foco. 2012. 3(4): 178181. Disponível em: http://biblioteca.cofen.gov.br/wpcontent/uploads/2016/02/Condicoes-detrabalho-de-enfermagem-e-adoecimento.pdf.

19. Silva RM. et al. Trabalho noturno e a repercussão na saúde dos enfermeiros. Revista Anna Nery. 2015. 15(2):270-376. Disponível em: https://www.scielo.br/scielo.php?pid=S1414$81452011000200008 \&$ script=sci_arttext.

20. Evangelista DS, Ribeiro, WA. Burnout syndrome and stress experienced by nurses in the intensive care unit: a literature review. Research, Society and Development. 2020. 7(9):1-23. Disponível em: https://www.redalyc.org/pdf/5057/505750895 011.pdf

21. Vidor $\mathrm{CR}$ et al. Prevalência de dor osteomuscular em profissionais de enfermagem de equipes de cirurgia em um hospital universitário. Acta Fisiatr. 2014. 21(1):6-10. Disponível em: https://www.revistas.usp.br/actafisiatrica/artic le/view/103819.

22. Neto AA et al. Qualidade de vida e nível de atividade física de profissionais de saúde de unidades de terapia intensiva. Revista Brasileira de Atividade Física \& Saúde. 2013. 18(6), 711-711. Disponível em: https://rbafs.org.br/RBAFS/article/view/2806.

23. Ozanam MAQ, et al. Satisfação e insatisfação no trabalho dos profissionais de enfermagem. Braz. J. of Develop. 2019. 5(6):6156-6178. Disponível em: https://www.brazilianjournals.com/index.php/ BRJD/article/view/1845.

24. Broca PV, Ferreira, MA. A comunicação da equipe de enfermagem de uma enfermaria de clínica médica. Rev Bras Enferm. 2018. 71(3):951-8. Disponível em: https://www.scielo.br/scielo.php?pid=S00347 $1672018000300951 \&$ script=sci_arttext\&tlng $=$ pt.

25. Azevedo IC et.al. Educação continuada em enfermagem no âmbito da educação permanente em saúde: revisão integrativa de literatura. Revista Em Saúde E Pesquisa. 2015.1(8):131-140. Disponível em: https://periodicos.unicesumar.edu.br/index.ph p/saudpesq/article/view/3275.

26. Salomé GM, et al. O ser profissional de enfermagem em Unidade De Terapia Intensiva. Acta Paulista de Enfermagem. 2018. 21(2):294-299. Disponível em: https://www.scielo.br/scielo.php?pid=S01032 $1002008000200010 \&$ script=sci_abstract\&tln $\mathrm{g}=\mathrm{pt}$.

27. Santos TVC, Penna CMM. Acessibilidade e resolutividade dos serviços de saúde: perspectivas de usuários e profissionais. Revista da faculdade de ciências gerenciais de manhuaçu. 2015. 12(1):98-108. Disponível em:

http://www.pensaracademico.facig.edu.br/ind ex.php/pensaracademico/article/view/213.

28. Moreira RM, Padilha KG. Ocorrências iatrogênicas com pacientes submetidos 
ventilação mecânica em unidade de terapia intensiva. Acta Paul Enferm. 2001. 14(2):918, 2001. Disponível em: https://actaape.org/en/article/ocorrencias-iatrogenicas- com-pacientes-submetidos-a-ventilacaomecanica-em-unidade-de-terapia-intensiva/.

Submissão: 2021-08-16

Aprovado: 2021-10-26 